\title{
Role of oxidised low density lipoprotein in atherogenesis
}

Joseph L Witztum

There can be little doubt that hypercholesterolaemia is a major risk factor for atherosclerosis. If plasma cholesterol concentrations rise above $160-180 \mathrm{mg} / \mathrm{dl}(4 \cdot 1-4 \cdot 7$ $\mathrm{mmol} / \mathrm{l}$ ) the risk of developing clinical coronary artery disease (CAD) increases proportionately. Recent studies have convincingly demonstrated that lowering raised plasma cholesterol can reduce this risk and even induce regression. However, at any given concentration of plasma cholesterol there is still great variability in the expression of clinical CAD. This diversity undoubtedly reflects complex and multifactorial events that are involved in the reactions of the artery to hypercholesterolaemia and the resulting atherogenesis accompanied by alterations in coagulation and vasomotor tone that result in clinical events. The fact that subjects with familial hypercholesterolaemia, who have profound increases in low density lipoprotein (LDL) concentrations from birth, nevertheless show considerable diversity in the expression of clinical disease clearly shows that factors other than raised LDL concentrations are involved in the atherogenic process and the clinical sequelae.

Clearly many factors are involved in atherogenesis and it is likely that in any given individual one or more of these factors may be relevant, but I propose to review the evidence that modifications of LDL, and specifically oxidative modification of LDL, are critically important and possibly even prerequisites for macrophage uptake and accumulation of cellular cholesteryl ester in the artery wall. In this review I will discuss the following four points.

- What is oxidised LDL?

- What is the evidence for the presence in vivo of oxidised LDL?

- What are the potential mechanisms by which oxidised LDL may be atherogenic?

- What measures may be taken to inhibit the oxidative modification process?

I hope that this review will convince the reader that sufficient evidence has been accumulated to suggest that clinical trials in humans to test the antioxidant process are currently indicated.

Medicine, University

of California, San

Diego, La Jolla,

California, USA

\section{J L Witztum}

Correspondence to

Dr Joseph L Witztum,

Department of Medicine 0682 , University of California,

San Diego, La Jolla, CA 92093-0682, USA

\section{What is oxidised LDL?}

The concept that modification of LDL is a prerequisite for macrophage uptake and cellular accumulation of cholesterol has been reviewed in detail. ${ }^{12}$ Here I will summarise the evidence that has led to this conclusion. $\stackrel{\mathbb{D}}{\circ}$ With the discovery by Brown and Goldstein कै of the LDL receptor pathway, it seemed $\overrightarrow{0}$ reasonable at first to suppose that this would $\rightarrow$ be the mechanism by which LDL was taken $\vec{\omega}$ up by macrophages - the cell type that chiefly accounts for the "foam cells" that typify the fatty streak. However, LDL incubated with macrophages in culture failed to have sufficient uptake to cause cholesteryl ester accumulation and furthermore, subjects (or $\frac{7}{0}$ rabbits) completely lacking LDL receptors is showed the most advanced atherosclerosis. $\vec{N}$ Therefore, another mechanism had to be 을 invoked. Again, it was Goldstein et al who $\overrightarrow{ }$ first proposed that modification of LDL was a prerequisite for macrophage uptake and cholesterol accumulation. ${ }^{3}$ They demonstrated $\underset{\gtrless}{\gtrless}$ that a chemical derivatisation of LDL, acety- $\vec{\theta}$ lation, led to enhanced macrophage uptake by a novel receptor, termed the scavenger receptor, and led to cholesteryl ester accumulation. Other chemically modified forms of LDL, such as malondialdehyde-conjugated LDL (MDA-LDL) were also recognised by the $\frac{}{\otimes}$ same receptor. Subsequent studies by Steinberg and colleagues showed that incuba- $\frac{9}{3}$ tion of LDL with cultured endothelial cells, or smooth muscle cells, converted LDL into a modified form now taken up more rapidly by macrophages. It was Steinbrecher et al ${ }^{4}$ who then showed that this was due to the ability of these cells to initiate lipid peroxidation in the LDL, a finding confirmed for smooth muscle cell modification as well. ${ }^{5}$ Important parallel studies showed that LDL was cytotoxic to 응 endothelial cells and smooth muscle cells and that the cytotoxicity was caused by oxidation of LDL lipids occurring during the incuba- N tion. ${ }^{6}$ It is now clear that oxidation of LDL can be induced by incubating it under appro- $N$ priate culture conditions with various cells, including endothelial cells, smooth muscle cells, macrophages, or even fibroblasts.

Oxidation can also be induced by incubation of LDL with a known catalyst of lipid peroxidation, such as copper or iron. ${ }^{4}$ The $\frac{0}{0}$ exact mechanisms by which cells initiate lipid $\stackrel{\mathbb{D}}{\mathbb{D}}$ peroxidation of LDL in the culture medium $\stackrel{\vec{\nabla}}{\mathbb{8}}$ have not been fully defined. It seems that several mechanisms may operate. For example, cellular lipoxygenases, such as 15-lipoxyge- o nase (15-LO), are likely to affect the ability of endothelial cells and macrophages to induce $\frac{\bar{a}}{\overrightarrow{2}}$ such modification, ${ }^{7}$ and superoxide anion may be secreted into the medium by cells such as smooth muscle cells. ${ }^{58}$ Once such an LDL is 
OPERATIONAL METHODS

$\downarrow$ ANTIOXIDANTS IN LDL (vitamin $E, \beta$-carotene, etc)

CHEMILUMINESCENCE, IODOMETRY

(methods may not be sensitive enough at this stage)

\section{$\uparrow$ ABSORBANCE AT $234 \mathrm{~nm}$ $\uparrow$ CHEMILUMINESCENCE $\uparrow$ PEROXIDES BY IODOMETRY}

$\downarrow$ POLYUNSATURATED FATTY ACID CONTENT

\section{$\uparrow$ FLUORESCENCE \\ $\uparrow$ T-BARS \\ $\uparrow$ IMMUNOREACTIVITY \\ (eg, anti-MDA-LDL)}

\section{$\downarrow$ RATE OF DEGRADATION BY FIBROBLASTS}

\section{$\uparrow$ RATE OF DEGRADATION BY MACROPHAGES}

Role of oxidised LDL in atherosclerosis and methods of detecting the changes at various stages of the process. (Reproduced with permission from the fournal of Clinical Investigation. ${ }^{2}$ )

\section{CHEMICAL EVENTS}
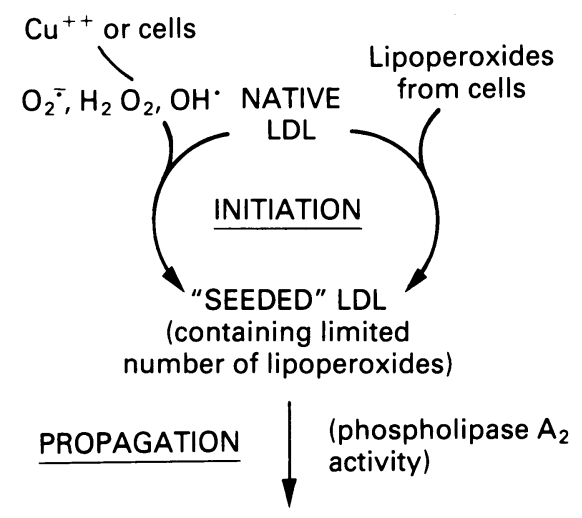

AMPLIFIED NUMBER OF

LIPOPEROXIDES IN LDL REARRANGEMENT OF DOUBLE BONDS

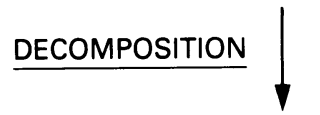

ALDEHYDES, KETONES FROM FATTY ACID FRAGMENTATION
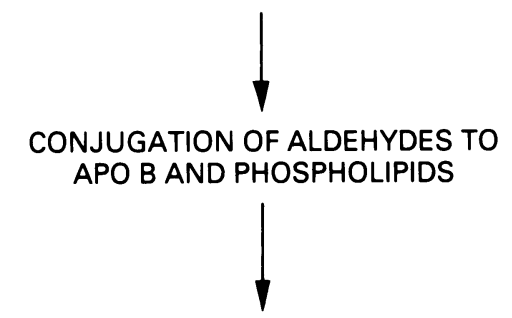
BY NATIVE LDL RECEPTOR

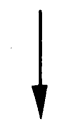

LDL RECOGNISED BY SCAVENGER RECEPTORS ("BIOLOGICALLY MODIFIED LDL")
LDL NO LONGER RECOGNISED

residues (and other amino acids as well) of apo $B$ inhibits the ability of LDL to bind to the LDL receptor, and when sufficient protein modification has occurred, this results in the generation of new epitopes on apo B that are now recognised by macrophage scavenger receptors. ${ }^{112}$ Freubis et al proposed that reactive fatty acid fragments may directly bind to apo $\mathrm{B}$ protein residues without the need for intermediate reactive aldehydes. ${ }^{13}$ Though the figure suggests an ordered series of reactions, in fact the complex reactions occur in a variable and even chaotic manner.

Consequently, it should be clear that oxidised LDL is not a single, homogeneous entity, but it is a particle containing heterogeneous products that include oxidised fatty acids and their breakdown products, oxidised sterols, oxidised phospholipids, as well as adducts of these various lipid products with apo $\mathrm{B}$ and phospholipids. In addition, some of these products may become quite polar and thus able to leave the LDL particle and mediate one or more of the biological effects that oxidised LDL has been reported to produce. In practical terms, it should be realised that oxidised LDL particles are quite heterogeneous, and furthermore, that the products formed from preparation to preparation may vary even when efforts are made to hold conditions of oxidation constant. These differences may depend on the cell types used to initiate oxidation, the metal ion concentrations, composition of the medium, the experimental conditions, and probably most importantly, the differences in inherent susceptibility to modification of different LDL preparations. A consequence of this is that various different effects of oxidised LDL on biological systems have been described, at times giving conflicting results between different laboratories. Such variability may be due in part to the fact that different components of the oxidised LDL may have different biological effects. This is discussed in further detail in our recent review, ${ }^{2}$ but this should serve to add a note of caution to the reader in interpreting the myriad of effects that oxidised LDL has now been reported to produce (see below).

We have assumed that the oxidative modification of LDL occurs primarily in the artery wall, in the intima, and most probably in microdomains sequestered from the many plasma antioxidants. It seems unlikely that significant degrees of oxidative modification of LDL can occur in plasma because of the many antioxidants present. Furthermore, were sufficient oxidation of LDL to occur in plasma, that oxidised LDL would be removed rapidly from plasma by scavenger receptors present on hepatic sinusoidal cells. However, plasma LDL could undergo limited oxidation which though it had no consequences for the LDL within the vascular space, might nevertheless lead to an LDL "primed" for more rapid oxidative modification in the intima. It seems likely that LDL is modified when LDL is on the surface of cells, such as endothelial cells or macrophages, or while bound to intiand other oxidised products, such as oxycholesterol derivatives. In part, these reactive intermediates may then complex with the adjacent apo B protein, as well as with phospholipids. The modification of the lysine




Table 1 Evidence for a role of oxidised $L D L$ in atherogenesis

(1) Epidemiological evidence showing an inverse relation between

dietary/plasma antioxidants and CAD.

(2) Immunocytochemica (2) Imusytochemical

vidence of oxidised LDL in

atherosclerotic lesions.

(3) Evidence that LDL

extracted from lesions has

physiochemical, immuno-

logical, and biological

properties of oxidised LDL

(4) Presence in serum of

autoantibodies with speci-

ficity for epitopes of oxidised LDI.

(5) Presence in lesions of IgG with specificity for epitopes of oxidised LDL. topes of oxidised LDL. 6) Presence in serum of subfractions of LDL with properties similar to early stages of oxidised LDL. (7) Ability of antioxidants to inhibit atherosclerosis in animal models.

Table 2 Potential mechanisms by which oxidised LDL has an impact on the atherogenic process

(1) By promoting macrophage cholesterylester enrichment through enhad untake through enhanced uptake through one or more scavenge receptors.

(2) By releasing products that are chemotactic for monocytes and $T$ lympho-

(3) By inhibiting the ability of macrophages to egress from the artery.

(4) By releasing products that are cytotoxic. (5) By altering gene expresinducing leucocyte adhesion molecules on monocytes and inducing synthesis of MCP 1 or II-1.

(6) By being immunogenic and potentially inducing humoral or cell mediated immunological responses. (7) By adversely affecting coagulation pathways, as for example by induction of tissue factor synthesis by macrophages.

(8) By adversely affecting (8) By adversely affecting coronary arteries. mal matrix, possibly in micro-sequestered loci created by extensions of cells, as demonstrated for macrophages by Heiple and colleagues. ${ }^{14}$

The implications of this argument are that it is primarily the antioxidant content of the LDL itself that is critical for its protection, and that the antioxidant environment of the intima and the ability of arterial cells to initiate lipid peroxidation may be the critical variables in determining the extent of arterial wall modification of LDL.

\section{Evidence of the presence in vivo of oxi- dised LDL}

Steinberg and I have reviewed in detail the evidence that oxidised LDL is present in vivo. ${ }^{2}$ Table 1 summarises the different lines of evidence that support this contention. First, epidemiological evidence of an inverse relation between dietary and/or plasma concentrations of antioxidants and the development of CAD is accumulating. Thus there is evidence that either levels of dietary intake or plasma concentrations or both of vitamin $E$, vitamin $C$ and $\beta$-carotene are inversely related to CAD. ${ }^{15-18}$ Furthermore, countries where consumption of monounsaturated fatty acids is high, with consequent enrichment of LDL with these fatty acids and more resistance to oxidative modification in general have lower rates of CAD. Second, immunocytochemical evidence, gained with antibodies to various epitopes on oxidised LDL showed intense staining of atherosclerotic lesions in both animals and humans but not of normal arterial tissue. ${ }^{19-21}$ Third, LDL extracted from lesions has been shown to have many of the physiochemical, immunological and, most importantly, biological properties attributed to in vitro preparations of oxidised LDL. ${ }^{22}$ Fourth, oxidised LDL is immunogenic and the presence in serum of animals and humans of autoantibodies with specificity for the epitopes of oxidised LDL has been demonstrated. ${ }^{20}$ In particular, a recent study of eastern Finnish men found that the titre of antibodies to an epitope of oxidised LDL was highly predictive of the subsequent progression of carotid artery atherosclerosis, implicating the oxidative process in atherogenesis. ${ }^{23}$ Fifth, in atherosclerotic lesions IgG with specificity for epitopes of oxidised LDL can be demonstrated, again suggesting the presence of oxidised LDL. Sixth, whereas extensive forms of oxidised LDL are not found in serum, several investigators have reported that plasma may contain small numbers of LDL particles that have the properties of LDL that has undergone the early steps of oxidative modification.

Though all of the above lines of evidence represent qualitative evidence that oxidised LDL is present in the artery wall, they do not provide information as to the quantitative importance of this process. However, if oxidation is important, then inhibiting it should inhibit the atherogenic process, and indeed, there are now numerous studies showing that lipophilic antioxidants, that are carried in the LDL core and which protect LDL as measured by in vitro assays, inhibit atherosclerosis in studies of hypercholesterolaemic rabbits and primates. Six different studies that used three different lipophilic antioxidants in hypercholesterolaemic rabbits showed inhibition of atherosclerosis ranging from 30 to $70 \%{ }^{24-29}$ Recently, probucol has been shown $\stackrel{\overline{\vec{S}}}{\stackrel{\overrightarrow{3}}{ }}$ to inhibit atherosclerosis in primates as well, independently of its ability to lower LDL concentrations. ${ }^{30}$ Furthermore, these effects could be shown to be independent of alterations in plasma LDL concentrations. The o pioneering studies of Carew et al ${ }^{24}$ clearly demonstrated that antioxidant therapy with probucol inhibited the uptake and degradation of LDL in portions of aorta containing atherosclerotic lesions, but not in portions of normal arteries. This is consistent with a direct effect of the antioxidants to prevent oxidative modification in macrophage-rich lesions. While it is conceivable that these $\frac{7}{0}$ antioxidants are inhibiting atherogenesis by as is yet undefined mechanisms unrelated to their $\vec{N}$ ability to protect LDL, it seems more likely 윽 that it is indeed their ability to protect LDL from oxidation that accounts for their ability to inhibit atherogenesis. Thus there are numerous lines of evidence strongly supporting the hypothesis that oxidative modification of LDL is an important factor, if not prerequisite, for mediating the atherogenicity of LDL.

Potential mechanisms by which oxidised LDL may have an impact on the atherogenic process (table 2)

Interest in the role of oxidised LDL began with the realisation that oxidised LDL had enhanced uptake in macrophages and promoted cholesteryl ester accumulation. However, it is now apparent that many other diverse biological effects may be induced by oxidised LDL. ${ }^{2}$ As noted above, many different products are generated during the oxidative modification of LDL and presumably each of these may have diverse biological effects. Thus products of oxidised LDL have been reported to be chemotactic for monocytes, which are widely regarded as the pre- ? cursors of most of the foam cells that form in $N$ the fatty streak. Potentially of equal impor- N tance is the recent realisation that up to $\geqslant 20 \%$ of the early cells found in the fatty 0 streak are $\mathrm{T}$ lymphocytes. Recent studies $\mathbb{D}$ have suggested that the products of oxidised : LDL are also chemotactic for this subclass of $\frac{T}{T}$ lymphocytes as well. A third potential mechanism is the fact that oxidised LDL inhibits migration of macrophages and thereby pre- $\stackrel{\mathbb{Q}}{\circ}$ vents their egress from the artery wall. One would ordinarily presume that once of macrophages had picked up toxic products, such as oxidised LDL, they would move out of the area and thereby restore it to normal. The fact that macrophages take up oxidised LDL but then remain may be an important event in the pathological accumulation of 
cholesterol in the artery wall. This may be worsened by the fact that products of oxidised LDL are highly cytotoxic to various cells, including endothelial cells. Disruption of endothelial cells after the formation of an intimal fatty streak has been demonstrated in experimental models, and the subsequent exposure to circulating blood elements, such as platelets, may be vital to the subsequent introduction into the lesion of growth factors, such as PDGF (platelet derived growth factor) that initiate the migration and proliferation of smooth muscle cells, which then convert the fatty streak into the more complicated lesion. Furthermore, the toxic effects of oxidised LDL, either from extracellular oxidised LDL in the intima, or possibly even from products within the macrophages filled with oxidised LDL, may initiate the cell death of macrophages, which typifies the more advanced lesion, leading to the frank deposition of "ceroid", the insoluble lipid that makes the gruel of the advanced lesion. Studies from my laboratory and others ${ }^{31}$ have shown that a component of ceroid is derived from oxidised LDL.

When LDL first enters the intimal space and is exposed to pro-oxidant conditions it undergoes the earliest steps of the oxidative supplement modification, and numerous products may be generated even though the LDL is not yet sufficiently modified so as to be recognised by the macrophage scavenger receptor. This so-called "minimally oxidised" or "minimally modified" LDL nevertheless may have an important role in the atherogenic process by releasing products that affect the gene expression in arterial cells. For example, such minimally oxidised LDL can stimulate the expression and secretion of $M$ CSF, GM-CSF, and G-CSF in human aortic endothelial cells, ${ }^{32}$ and when injected in vivo in mice, caused a 7-26 fold increase in serum M-CSF activity. ${ }^{33}$ Such minimally oxidised LDL can stimulate monocyte-specific adherence molecules on the surface of endothelial cells, thereby leading to yet another mechanism for monocyte chemoattraction, and, importantly, can stimulate the secretion of monocyte chemotactic protein-1 (MCP-1) by cultured human aortic, endothelial, and smooth muscle cells ${ }^{34}$ and when injected in vivo in mice can increase the mRNA expression of JE (the mouse homologue of MCP-1) in liver and other tissues. ${ }^{33}$ Using in situ hybridisation techniques, we have demonstrated that MCP-1 and M-CSF message are found in macrophage-rich regions of both rabbit and human aortas but not in normal aortas. ${ }^{3536}$ Thus it is likely that oxidised LDL can induce arterial wall cells to produce various potent chemotactic factors, adhesion molecules, cytokines, and growth factors that have an important role in development and maturation of the atherosclerotic plaque. Though we do not know at present which of the many different products generated during the oxidative modification of LDL are responsible for these different effects, this is an exciting area for future research that undoubtedly will have important long-term consequences.

We have previously shown that even minor modifications of LDL are immunogenic and that oxidised LDL is immunogenic too. Autoantibodies against various epitopes of oxidised LDL are present in human serum, and immunoglobulin specific for epitopes of oxidised LDL can be found in atherosclerotic lesions. Whether or not these antibodies are mere epiphenomena reflecting an immune response to altered proteins and lipids is at present unknown, but it is of interest that the titre of autoantibody to an epitope of oxidised LDL, MDA-lysine, was an effective predictor of the progression of carotid atherosclerosis in a recent study.$^{23}$ Since many lines of evidence suggest that there is activation of immune cells within the artery wall, the presence of specific immune complexes could provide at least one mechanism by which activation of $T$ cells could occur, resulting in cell-mediated immunological responses. Since $\mathrm{T}$ cells are clearly an important component of the early fatty streak lesion, this is an area that we need to know much more about (reviewed in reference 37).

Finally, oxidised LDL may alter two vital properties that result in the often fatal sequelae that mark the atherosclerotic artery. As outlined elsewhere in this supplement, it is clear that thrombotic events can occur on lesions that are only modestly involved with atherosclerotic events and that plaque rupture and initiation of thrombosis can occur at the lateral margins of such lesions. In this regard, the observation that macrophages containing oxidised LDL are also found in such regions is of potential importance, particularly in light of the recent report that oxidised LDL may stimulate the release of tissue factor by macrophages. ${ }^{38}$ In addition, there is now an extensive body of information suggesting that hypercholesterolaemia in itself may impair the EDRF-mediated vasorelaxation of coronary arteries in response to agents, such as acetylcholine. In large part, this inhibition seems to be mediated via products of oxidised LDL, either through direct disruption of receptormediated signalling to the nitric oxide synthase, or by interfering with the nitric oxide (or nitric oxide/thiol adduct) that is the EDRF factor (see reference 39). Thus hypercholesterolaemia in itself, by generating more oxidised LDL in the arterial intima, may alter vasomotor responses, even producing paradoxical vasoconstriction in respose to normal vasodilatatory stimuli, and contribute importantly to vasospasm, even in the absence of significant lesions. Of course, this may be greatly accentuated in those areas where fatty streaks or intermediate lesions are present. Thus, lowering cholesterol and inhibiting oxidation may have profound effects on the clinical events marking CAD, even without causing major changes in arterial wall structure.

In summary, while it remains to be proved that all or even some of these phenomena have in vivo counterparts, the concept is 
clearly emerging that oxidised LDL may contribute importantly to the atherogenic process via mechanisms above and beyond that of contributing to cholesterol deposition in the placque and narrowing of the vessel lumen. In fact, it is possible that oxidised LDL may promote the atherogenic process by virtue of its oxidation products, even before or coincident with the delivery of lipid to the arterial cells. This implies that factors that inhibit the oxidation of LDL may have even more profound effects on inhibiting the atherogenic process and the resulting clinical events than can be measured simply by inhibition of plaque formation.

\section{Measures to inhibit oxidative modifica- tion of LDL}

One can approach the subject of inhibition of the oxidation of LDL in vivo by understanding those factors that lead to this process. First, we must consider factors intrinsic to the LDL particle itself. The nature of the substrate for lipid peroxidation, mainly the polyunsaturated fatty acids in lipid esters and cholesterol, is a dominant influence in determining susceptibility. As noted by Esterbauer et al there is a vast excess of polyunsaturated fatty acids in LDL, chiefly in the form of linoleic acid, in relation to the content of natural, endogenous antioxidants. ${ }^{9}$ The importance of the fatty acid composition has been demonstrated by recent studies in our laboratory in rabbits and in humans that showed that increasing the oleic acid content of LDL, at the expense of linoleic acid, will greatly protect the LDL from oxidation. ${ }^{40-42}$ Secondly, the endogenous content of natural antioxidants, including vitamin $\mathrm{E}, \beta$-carotene, ubiquinol-10, and others (as well as the presence of exogenously administered antioxidants such as probucol) are exceedingly important. Studies by Esterbauer et al clearly show that these endogenous antioxidants must be consumed before the initiation of the rapid propagation phase of lipid peroxidation. ${ }^{9} \mathrm{~A}$ third factor that seems to affect the susceptibility of LDL to oxidation is particle size-with smaller, more dense LDL subfractions displaying an apparent increased propensity to be oxidised. Theoretically, differences in the intrinsic phospholipase $\mathbf{A}_{2}$ activity, which greatly promotes propagation reactions, may also be an important determinant.

In addition to the inherent susceptibility of the given LDL particle, factors extrinsic to LDL that promote oxidation also may be vitally important. Such factors may include potential variation in cellular pro-oxidant activity, as for example differences in the ability of macrophages to modify LDL because of inherent differences in macrophage expression of 15-lipoxygenase or the ability of cells to secrete cellular superoxide anion or thiols. ${ }^{4344}$ Secondly, the concentration of plasma and extracellular fluid peroxidant components, such as trace metal concentrations, may be involved, as is the concentration of binding proteins that complex with these metals. A third possibility is the concentration of plasma and extracellular fluid antioxidant compounds such as urate $w$ and importantly, ascorbate. Though ascorbate is hydrophilic, nevertheless it is capable of converting the alpha tocopherol radical ? (generated when vitamin $\mathrm{E}$ acts as an antioxidant) to reduced alpha tocopherol, thereby $\stackrel{\vec{S}}{\vec{S}}$ recycling it to act again as an antioxidant. ${ }^{945} \mathrm{O}$ Presumably, at the interface between an LDL 흠 particle and ascorbate, such a reaction could $\frac{\bar{\sigma}}{7}$ help to maintain an effective vitamin $\mathrm{E}$ con- $\stackrel{\mathbb{Q}}{\Omega}$ centration in an LDL particle. HDL has also been shown to inhibit the oxidation of LDL $\vec{\circ}$ in cell culture ${ }^{46}$ and in a model system, ${ }^{47}$ though the mechanisms for this property $\vec{\omega}$ remain unknown. Finally, factors influencing the residence time of LDL in the intima are undoubtedly very important as well-that is, 8 factors that increase binding of LDL such as $\mathrm{Lp}(\mathrm{a})$, differences in localised matrix proteins $\stackrel{\mathcal{C}}{\mathrm{C}}$ and glycoproteins that bind LDL, or pro- $\frac{0}{0}$ cesses such as non-enzymatic glycosylation of $\bar{c}$ LDL or matrix, all of which could prolong $\vec{N}$ the residence of LDL in the intima and there- 을 by increase the potential for it to be oxidised.

From the discussion above it follows that interventions to inhibit oxidative modification can be thought of as being focused in these two general areas: measures to protect LDL $\vec{\bullet}$ itself and measures to reduce those factors $\stackrel{\oplus}{\omega}$ responsible for initiating oxidation of $\mathrm{LDL}$. At present, probucol is the most potent lipophilic antioxidant capable of protecting LDL. Its demonstrated ability to inhibit atherosclerosis in rabbits, despite its ability to $\frac{}{\otimes}$ lower HDL, suggests that it is a prime candi- $\stackrel{\varrho}{\Rightarrow}$ date to use in testing the antioxidant hypothesis in humans. Recent data from our laboratory in which oleic acid enrichment of LDL was achieved at the expense of linoleic acid shows the principle of this type of dietary intervention to inhibit the oxidative process, ${ }^{40-42}$ but the extent to which such pro- 3 tection can be achieved in free-living populations remains to be fully demonstrated. Though the studies used extreme changes in 0 diet, it nevertheless seems reasonable to believe that moderate increases in mono- 을 unsaturated fatty acids, at the expense of polyunsaturated fatty acids, while producing moderate changes, may have important clini- $N$ cal effects when integrated over long periods. N

Supplementation with natural lipophilic antioxidants, such as vitamin $\mathrm{E}$ and $\beta$ carotene, also seems appropriate. Indeed, in studies in which human subjects were supplemented with vitamin $\mathrm{E}$ it was shown that the vitamin $\mathrm{E}$ content of LDL was enriched two $\underset{\mathbb{D}}{\mathbb{D}}$ to three fold, and these LDLs were signifi- $\frac{\Omega}{\mathbb{D}}$ cantly less susceptible to oxidative stress. $^{9} \frac{O}{\circ}$ Theoretically $\beta$-carotene should produce a similar effect, but there are no data, at least in $\frac{8}{0}$ vivo, to show that $\beta$-carotene feeding will protect LDL. It must be recognised that antioxi- $\frac{0}{7}$ dants, such as probucol or vitamin $\mathrm{E}$, may also have important effects in inhibiting the ability of cells to modify LDL, as recently demonstrated by Parthasarathy using a water- 
soluble form of probucol. ${ }^{48}$ While dietary supplementation with the natural antioxidant vitamins, such as $E$ and $\beta$-carotene (and presumably other carotenoids) seems reasonable, particularly because there are no concerns about the side effects, as yet there are no prospective data on their effectiveness in vivo. Combinations of these interventions could prove synergistic, like the dietary supplementation of ascorbic acid noted above. Such interventions are currently feasible and intensive study is now underway to determine the optimal forms of intervention to protect LDL. A recent consensus conference supported the notion that sufficient evidence was available to encourage intervention trials in humans to test this hypothesis. ${ }^{49}$ It seems reasonable to use probucol and a vitamin cocktail (for example, containing vitamin $\mathrm{E}$, vitamin $C$ and $\beta$-carotene) or both to test the antioxidant hypothesis in humans.

On the other hand experimental approaches designed to inhibit the ability of cells to modify LDL are more theoretical at present, but may offer alternative approaches as well. For example, if it can be demonstrated that inhibition of macrophage 15-lipoxygenase activity in animal models inhibits atherosclerosis, then this would offer a novel approach that would act synergistically with measures directly to protect the LDL itself. Finally, it should be noted that perhaps the most effective way to inhibit the oxidative modification of LDL is to reduce plasma LDL concentrations, thereby reducing the substrate in a most direct manner.

1 Steinberg D, Parthasarthy S, Carew TE, Khoo JC, Witztum JL. Beyond cholesterol: Modifications of low density lipoprotein that increase its atherogenicity. $N$ Engl f Med 1989;320:915-24.

2 Witztum JL, Steinberg D. Role of oxidized LDL in atherogenesis. 7 Clin Invest $1991 ; 88: 1785-92$

3 Goldstein JL, Ho YK, Basu SK, Brown MS. Binding site on macrophages that mediates uptake and degradation of acetylated low density lipoprotein, producing massive
cholesterol deposition. Proc Natl Acad Sci USA 1979; cholesterol

4 Steinbrecher UP, Parthasarathy S, Leake DS, Witztum JL, Steinberg D. Modification of low density lipoprotein by endothelial cells involves lipid peroxidation and degradation of low density lipoprotein phospholipids. Proc Natl Acad Sci USA 1984;81:3883-7.

5 Heinecke JW, Rosen H, Chait A. Iron and copper promote modification of low density lipoprotein by human arterial smooth muscle cells in culture. $f$ Clin Invest 1984;74:1890-4.

6 Morel DW, DiCorleto PE, Chisholm GM. Endothelial and smooth muscle cells alter low density lipoprotein in vitro by free radical oxidation. Arteriosclerosis 1984;4: 357-64

7 Rankin SM, Parthasarathy S, Steinberg D. Evidence for a dominant role of lipoxygenase(s) in the oxidation of LDL by mouse peritoneal macrophages. F Lipid Res 1991;32:449-56.

8 Steinbrecher UP. Role of superoxide in endothelial-cell modification of low-density lipoproteins. Biochim Biophys Acta 1988;959:20-30.

9 Esterbauer H, Gebicki J, Puhl H, Jürgens G. The role of lipid peroxidation and antioxidants in oxidative modifilipid peroxidation and antioxidants in oxidative mo.

10 Parthasarathy S, Steinbrecher UP, Barnett J, Witztum JL, Steinberg $D$. Essential role of phospholipase $A_{2}$ activity
in endothelial cell-induced modification of low density in endothelial cell-induced modification of low density

11 Steinbrecher UP, Witztum JL, Parthasarathy S, Steinberg $D$. Decrease in reactive amino groups during oxidation or endothelial cell modification of LDL: correlation with changes in receptor-mediated catabolism. Arteriosclerosis 1987:11:35-143.

12 Parthasarathy S, Fong LG, Otero D, Steinberg D Recognition of solubilized apoproteins from delipidated oxidized low density lipoprotein (LDL) by the acetylLDL receptor. Proc Natl Acad Sci USA 1987;84:537-40.

13 Freubis J, Parthasarathy S, Steinberg D. Evidence for a polypeptides. Proc Natl Acad Sci USA (in press).
14 Heiple JM, Wright SD, Allen NS, Silverstein SC. Macrophages form circular zones of very close apposi-
tion to IgG-coated surfaces. Cell Motil Cytoskeleton tion to IgG-coate

15 Manson JE, Stampfer MJ, Willett WC, et al. A prospective study of antioxidant vitamins and incidence of coronary heart disease in women. Circulation 1991;84:546.

16 Gaziano JM, Manson JE, Branch LG, et al. Dietary beta carotene and decreased cardiovascular mortality in an elderly cohort [abstr]. $\mathcal{F} \mathrm{Am}$ Coll Cardiol 1992;19:377.

17 Gey KF, Puska P. Plasma vitamins E and A inversely correlated to mortality from ischemic heart disease in crosscultural epidemiology. Ann NYAcad Sci 1989;570: 268-82.

18 Enstrom JE, Kanim LE, Klein MA. Vitamin C intake and mortality among a sample of the United States population. Epidemiology 1992;3:194-202.

19 Haberland ME, Fong D, Cheng L. Malondialdehydealtered protein occurs in atheroma of Watanabe heritable hyperlipidemic rabbits. Science 1988;242:215-8.

20 Palinski W, Rosenfeld ME, Ylä-Herttuala S, et al. Low density lipoprotein undergoes oxidative modification in vivo. Proc Natl Acad Sci 1989;86:1372-6.

21 Boyd HC, Gown AM, Wolfbauer G, Chait A. Direct evidence for a protein recognised by a monoclonal antibody against oxidatively modified LDL in atherosclerotic lesions from a Watanabe heritable hyperlipidemic otic lesions from a Watanabe heritab
rabbit. Am f Pathol 1989;135:815-25.

22 Ylä-Herttuala S, Palinski W, Rosenfeld ME, et al. Evidence for the presence of oxidatively modified low density lipoprotein in atherosclerotic lesions of rabbit and man. $\mathcal{F}$ Clin Invest 1989;84:1086-95.

23 Salonen JT, Ylä-Herttuala S, Yamamoto $R$, et al. Autoantibody against oxidised $\mathrm{LDL}$ and progression of carotid atherosclerosis. Lancet 1992;339:883-7.

24 Carew TE, Schwenke DC, Steinberg D. Antiatherogenic effect of probucol unrelated to its hypocholesterolemic effect: evidence that antioxidants in vivo can selectively inhibit low density lipoprotein degradation in macrophage-rich fatty streaks and slow the progression of atheroslcerosis in the Watanabe heritable hyperlipidemic rabbit. Proc Natl Acad Sci USA 1987;84:7725-9.

25 Kita T, Nagano Y, Yokode $M$, et al. Probucol prevents the progression of atherosclerosis in Watanabe heritable hyperlipidemic rabbit, an animal model for familial hypercholesterolemia. Proc Natl Acad Sci USA 1987;84:5928-31.

26 Daugherty A, Zweifel BS, Schonfeld G. Probucol attenutates the development of aortic atherosclerosis in cholesterol-fed rabbits. Br ₹ Pharmacol 1989;98:612-8.

27 Bjorkhem I, Henriksson-Freyschuss A, Breuer $\mathrm{O}$, et al, The antioxidant butylated hydroxytoluene protects against atherosclerosis. Arterio Thromb 1991;11:15-22.

28 Mao SJ, Yates MT, Parker RA, et al. Attenuation of atherosclerosis in a modified strain of hypercholesatherosclerosis in a modified strain of hypercholesterolemic Watanabe rabbits with use of a probucol ana-
logue that does not lower serum cholesterol. Arterioscl logue that does not lower
Thromb 1991;11:1266-75.

29 Sparrow CP, Doebber TW, Olszewski J, et al. Low density lipoprotein is protected from oxidation and the progression of atherosclerosis is slowed in cholesterol-fed rabbits by the antioxidant $\mathrm{N}, \mathrm{N}^{\prime}$-dephenyl-phenylenediamine. $\mathcal{F}$ Clin Invest 1992;89:1885-91.

30 Ross R. Personal communication.

31 Parums DV, Brown DL, Mitchinson MJ. Serum antibodies to oxidized low density lipoprotein and ceroid in chronic periaortitis. Arch Pathol Lab Med 1990;114: chronic

32 Rajavashisth TB, Andalibi A, Territo MC, et al. Induction of endothelial cell expression of granulocyte and macrophage colony-stimulating factors by modified low density lipoproteins. Nature 1990;344:254-7.

33 Liao F, Berliner JA, Mehrabian M, et al. Minimally modified low density lipoprotein is biologically active in vivo. f Clin Invest 1991;87:2253-7.

34 Cushing SD, Berliner JA, Valente AJ, et al. Minimally modified low density lipoprotein induces monocyte chemotactic protein 1 in human endothelial cells and smooth muscle cells. Proc Natl Acad Sci USA 1990;87: 5134-8.

35 Ylä-Hertuala S, Lipton BA, Rosenfeld ME, et al. Macrophages express monocyte chemotactic protein Macrophages express monocyte chemotactic protein Proc Natl Acad Sci USA 1991;88:5252-6.

36 Rosenfeld ME, Ylä-Herttuala S, Lipton BA, Ord VA, Witztum JL, Steinberg D. Macrophage colony-stimulating factor mRNA and protein in atherosclerotic lesions of rabbits and man. Am $\mathcal{f}$ Pathol 1992;140:291-300.

37 Libby P, Hansson GK. Involvement of the immune system in human atherogenesis: current knowledge and unanswered questions. Lab Invest 1991;64:5-15.

38 Drake TA, Hannani K, Fei HH, Lavi S, Berliner JA. Minimally oxidized low-density lipoprotein induces tissue factor expression in cultured human endothelial cells. Am $¥$ Pathol 1991;138:601-7.

39 Rosenfeld ME. Oxidized LDL affects multiple atherogenic cellular responses. Circulation 1991;83:2137-40.

40 Parthasarathy S, Khoo JC, Miller E, Barnett J, Witztum JL, Steinberg D. Low density lipoprotein enriched in oleic acid is protected against oxidative modification: oleic acid is protected against oxidative modification: Implications for dietary prevention of a

41 Reaven P, Parthasarathy S, Grasse BJ, et al. Feasibility of using an oleate-rich diet to reduce the susceptibility of 
low density lipoprotein to oxidative modification in humans. Am f Clin Nutr 1991;54:701-6.

42 Reaven P, Parthasarathy S, Grasse BJ, Miller E, Steinberg $D$ Witztum JL. Effects of oleate-rich and linoleate-rich diets on the susceptibility of low density lipoprotein to oxidative modification in mildly hypercholesterolemic subjects. $f$ Clin Invest (in press). 3 subjects ${ }^{2}$, Rin Invest (in press).

sule of sulfur-containing amino acids in superoxide production and modification of low density lipoprotein by arterial smooth muscle cells. f Biol Chem 1987;262:10098-103.

44 Parthasarathy $S$. Oxidation of low-density lipoprotein by thiol compounds leads to its recognition by the acetyl LDL receptor. Biochim Biophys Acta 1987;917:337-40.

45 Jialal I, Vega GL, Grundy SM. Physiologic levels of ascorbate inhibit the oxidative modification of low density lipoprotein. Atherosclerosis 1991;82:185-91.
46 Parthasarathy S, Barnett J, Fong LG. High density lipoprotein inhibits the oxidative modification of low density lipoprotein. Biochim Biophys Acta 1990; 1044:275-83.

47 Navab M, Imes SS, Hama SYet al. Monocyte transmigration induced by modification of low density lipoprotein in cocultures of human aortic wall cells is due to induction of monocyte chemotactic protein 1 synthesis and is abolished by high density lipoprotein. $\mathcal{f}$ Clin Invest 1991;88:2039-46.

48 Parthasarathy S. Evidence for an additional intracellular site of action of probucol in the prevention of oxidative modification of low density lipoprotein: Use of a new water-soluble probucol derivative. $\mathcal{f}$ Clin Invest 1992;89: 1618-821.

49 Steinberg and Workshop Participants. Antioxidants in the prevention of human atherosclerosis. Circulation 1992;85:2337-44. 